\title{
PRIMER REGISTRO DE Etiella zinckenella (Treitschke) (Lepidoptera, Pyralidae) AFECTANDO SEMILLAS DE Alstroemeria spp. NATIVAS EN VALPARAÍSO, CHILE
}

\section{FIRST RECORD OF Etiella zinckenella (Treitschke) (Lepidoptera, Pyralidae) AFFECTING SEEDS OF NATIVE Alstroemeria spp. PLANT IN VALPARAÍSO, CHILE}

\author{
Jimena Ampuero $^{1 \mathrm{a} *}$, Daniela León ${ }^{1 \mathrm{~b}}$ \\ ${ }^{1 a}$ Escuela de Ciencias Agrícolas y Veterinarias, Universidad Viña del Mar, Agua Santa 7055, sector \\ Rodelillo, Viña del Mar, Chile \\ https://orcid.org/0000-0002-3605-7039 \\ ${ }^{1 b}$ Escuela de Ciencias Agrícolas y Veterinarias, Universidad Viña del Mar, Agua Santa 7055, sector \\ Rodelillo, Viña del Mar, Chile \\ 0000-0002-0947-9359 \\ * Autor para correspondencia E-mail: jampuero@uvm.cl
}

\section{RESUMEN}

Etiella zinckenella es una plaga cosmopolita de distribución mundial que ataca principalmente a especies de la familia Fabaceae, considerándose la soya como su principal hospedero. La larva del insecto ingresa a las vainas y se alimenta de las semillas, produciendo fecas que se acumulan y generan la pérdida de las semillas. En Chile, se ha reportado la presencia de E. zinckenella en la Región Metropolitana, sin especificar el hospedero en el cual se detectó. Durante el período febrero-marzo de 2019, se prospectaron poblaciones de Alstroemeria spp. nativas (A. angustifolia, A. cummingiana y A. marticorenae) en el sector de Rodelillo Alto, Viña del Mar, para la colecta de frutos con la finalidad de identificar el agente causal de perforaciones detectadas en éstos. En las cápsulas recolectadas se registró la presencia de larvas de E. zinckenella alimentándose de las semillas. La determinación del insecto a nivel de especie se realizó con un análisis morfológico de las larvas, ratificado a través de la observación de genitalias de hembras y machos adultos obtenidos en crianza. En este estudio se documenta la primera detección e identificación de E. zinckenella en poblaciones de Alstroemerias nativas en Chile.

Palabras clave: Pyralidae, Phycitinae, Alstromeriaceae.

\section{ABSTRACT}

Etiella zinckenella is a cosmopolitan pest of worldwide distribution that mainly attacks species of the Fabaceae family, particularly soybean as it is considered the main host for the insect. The larvae enter the pods and feed on the seeds, producing feces that accumulate and cause seed loss. In Chile, E. zinckenella has been reported in the Metropolitan Region, but there is no information about the host in which it was detected. During the period February-March 2019, populations of native Alstroemeria spp. (A. angustifolia, A. cummingiana and A. marticorenae) were surveyed in the sector of Rodelillo Alto, Viña del Mar, and fruits were collected in order to identify the causal agent of perforations detected in them. In the capsules collected, the presence of E. zinckenella larvae feeding on the seeds was recorded. The determination of the insect at the species level was carried 
out with a morphological analysis of the larvae, confirmed through observation of the genitalia of adult females and males during rearing. This study is the first report on detection and identification of E. zinckenella in native Alstroemerias populations in Chile.

Key words: Pyralidae, Phycitinae, Alstromeriaceae.

\section{INTRODUCCIÓN}

La familia Pyralidae, orden Lepidoptera, tiene amplia distribución en el mundo y agrupa a más de 5.900 especies incluidas en 1.055 géneros. Los adultos de esta familia son de tamaño pequeño a mediano (10 a $25 \mathrm{~mm})$, aunque algunas especies pueden superar los $30 \mathrm{~mm}$. La cabeza está cubierta por escamas anchas apretadas, la probóscide presenta escamas en su base y los palpos labiales son prominentes. Las alas anteriores son subtriangulares o alargadas y las alas posteriores son anchas. En reposo las alas permanecen como techo o envuelven el cuerpo, mientras que las antenas se dirigen hacia atráss sobre éste (Urra, 2018).

En Chile, la subfamilia Phycitinae registra un total de 16 géneros, los que adscriben un total de 21 especies (Cepeda, 2017). Las especies pertenecientes a esta sub familia, usualmente son de tamaño pequeño, con las alas anteriores estrechas, chaetosemata presente y frenulum con una sola cerda. En la genitalia del macho, el uncus posee ramas extendidas en ángulo obtuso y el tegumen conectado por una estrecha sección a la base del uncus; en la genitalia de la hembra, por lo general, el ductus seminalis se origina del corpus bursae. En la larva, la seta SD1 del mesotórax tiene una esclerotización circular (Cepeda, 2017).

Las larvas generalmente son enrolladoras, en que algunas de ellas elaboran capullos mezclados con fecas. Otras, son perforadoras de yemas, brotes, frutos, semillas o desarrollan galerías en sus hospederos (Zhang, 2011; Prayogo and Bayu, 2020).

El género Etiella (Zeller, 1839) (Pyralidae: Phycitinae), contiene especies cuyas larvas se alimentan principalmente de semillas de fabáceas. La especie Etiella zinckenella, de distribución cosmopolita, es una plaga de interés económico en la producción de soya (Kuswantoro et al., 2017; Prayogo and Bayu, 2020). En Chile, E. zinckenella se distribuye en la Región Metropolitana, Provincia de Santiago (Cepeda, 2017). Sin embargo, no se indica la o las especies vegetales en que se ha observado la plaga. El objetivo de este trabajo fue documentar la primera detección e identificación de este insecto en poblaciones de Alstroemerias nativas en el sector de Rodelillo Alto, Valparaíso.

\section{MATERIALES Y MÉTODOS}

Durante los meses de febrero-marzo de 2019 se prospectaron poblaciones de Alstroemeria spp. nativas ( $A$. angustifolia, $A$. cummingiana y $A$. marticorenae), ubicadas en el sector de Rodelillo

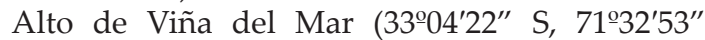
W) distribuidas en un sector con una superficie de 13 ha, con la finalidad de colectar sus frutos (cápsulas) (Fig. 1).

Los frutos colectados se ubicaron en placas de Petri y se mantuvieron a temperatura ambiente $\left(21-23^{\circ} \mathrm{C}\right)$ durante dos semanas, momento en el que se detectó la emergencia de larvas desde un $30 \%$ de las cápsulas almacenadas (Fig. 2). Las placas con presencia del insecto se mantuvieron intactas, a temperatura ambiente durante 60 días, para realizar seguimiento a la evolución en el desarrollo de las larvas. La identificación a nivel de especie se realizó con el uso de clave de estados inmaduros de lepidóptera (Artigas, 1994; Solis, 2006) y se ratificó a través de la preparación de la genitalia de los machos y hembras emergidos, la cual se comparó con la descripción realizada por Whalley (1973).

Las fotografías se tomaron con cámara (Sony $\alpha$ 5100, Bangkok, Tailandia) adaptada a un microscopio (Olympus BX41, Tokio, Japón) y el mapa de localización se realizó con Simplemappr (www.simplemappr.net).

\section{RESULTADOS Y DISCUSIÓN}

La morfología de las larvas detectadas en semillas de plantas de Alstroemeria spp. concuerdan con lo descrito por Artigas (1994) para larvas de Etiella zinckenella, presentando desde el tercer al sexto segmento abdominal tres setas ventrales, espiráculo del octavo segmento abdominal al mismo nivel que los precedentes, un anillo brillante alrededor de la cerda SD1 en el octavo segmento abdominal, con tres cerdas en el grupo lateral del noveno segmento abdominal y placa torácica con patrón característico (Fig. 3A).

Así mismo, la genitalia del macho presenta procesos costales en valvas largos, yuxta con dos lóbulos laterales anchos y elongados, redondeados en el ápice. Edeago con cornuti esclerotizado y con numerosas espinas (Fig. 3B y C). Las hembras presentan ductus bursae largo, esclerotizado, estrechándose a medida que se abre hacia la 


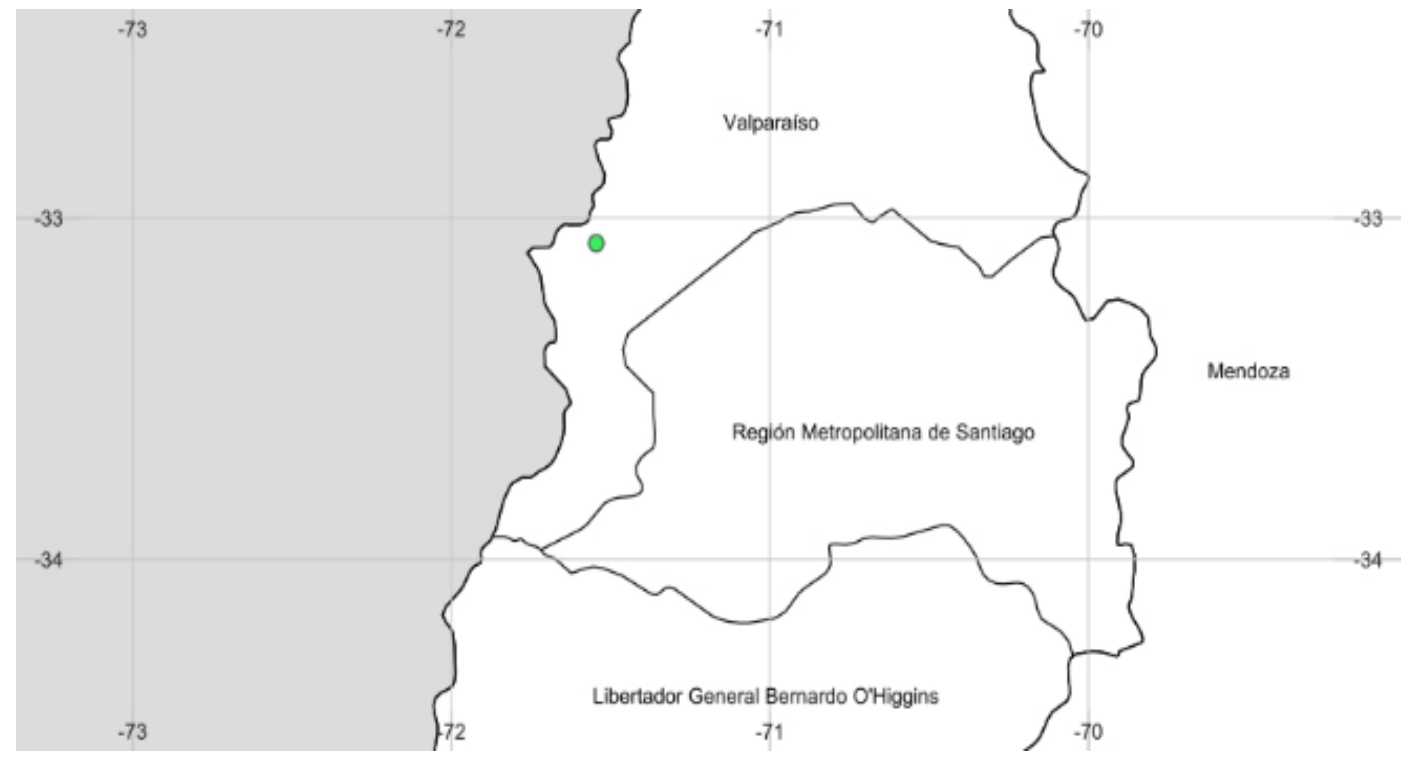

Fig. 1. Zona de colecta de cápsulas de Alstroemeria spp. Rodelillo Alto, Valparaíso, Región de Valparaíso, Chile.

Fig. 1. Area of collection of Alstroemeria spp. capsules, Rodelillo Alto, Valparaíso, Valparaíso Region, Chile.

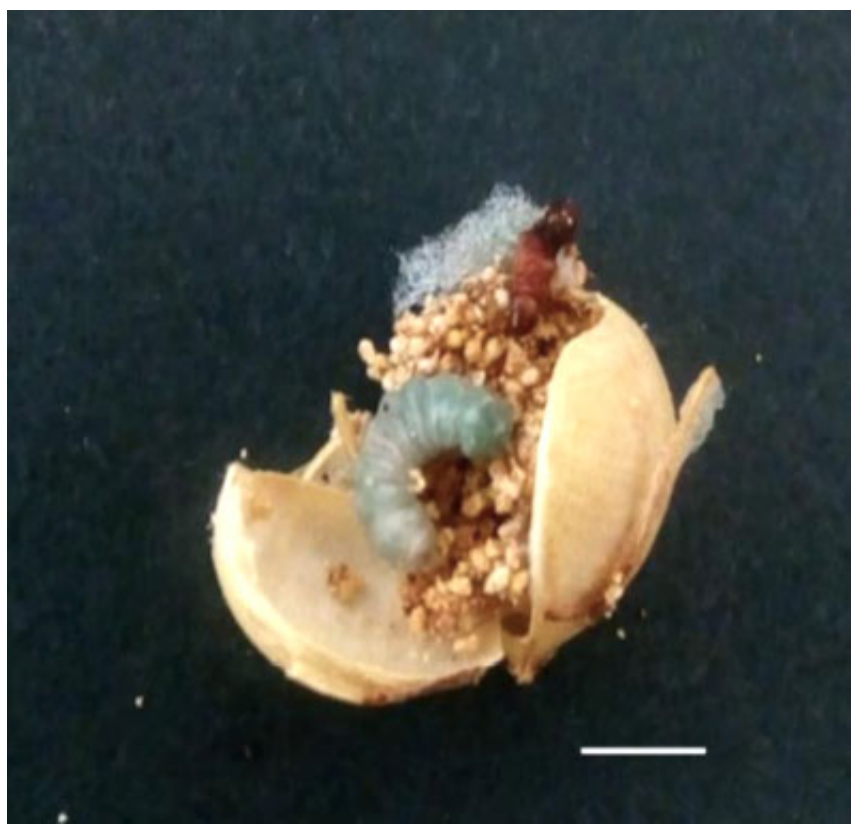

Fig. 2. Daño de larva E. zinckenella en cápsula de Alstroemeria sp. (Barra escala 0,5 cm).

Fig. 2. Damaged caused by larvae of E. zinckenella in Alstroemeria sp. capsules (Scale bar $0.5 \mathrm{~cm}$ ).

bursa. Saco secundario grande, esclerotizado, con espinas. Signum en bursa principal con espinas largas (Fig. 3D). Las características morfológicas de ambas genitalias coinciden con lo descrito por Whalley (1973) para la especie E. zinckenella.

Tanto las larvas como los adultos y genitalias de los mismos se encuentran depositados en la colección entomológica de la Escuela de Ciencias Agrícolas y Veterinarias de la Universidad de Viña del Mar.

Material examinado: Chile, Región de Valparaíso, Sector Rodelillo, II-2019, Daniela 


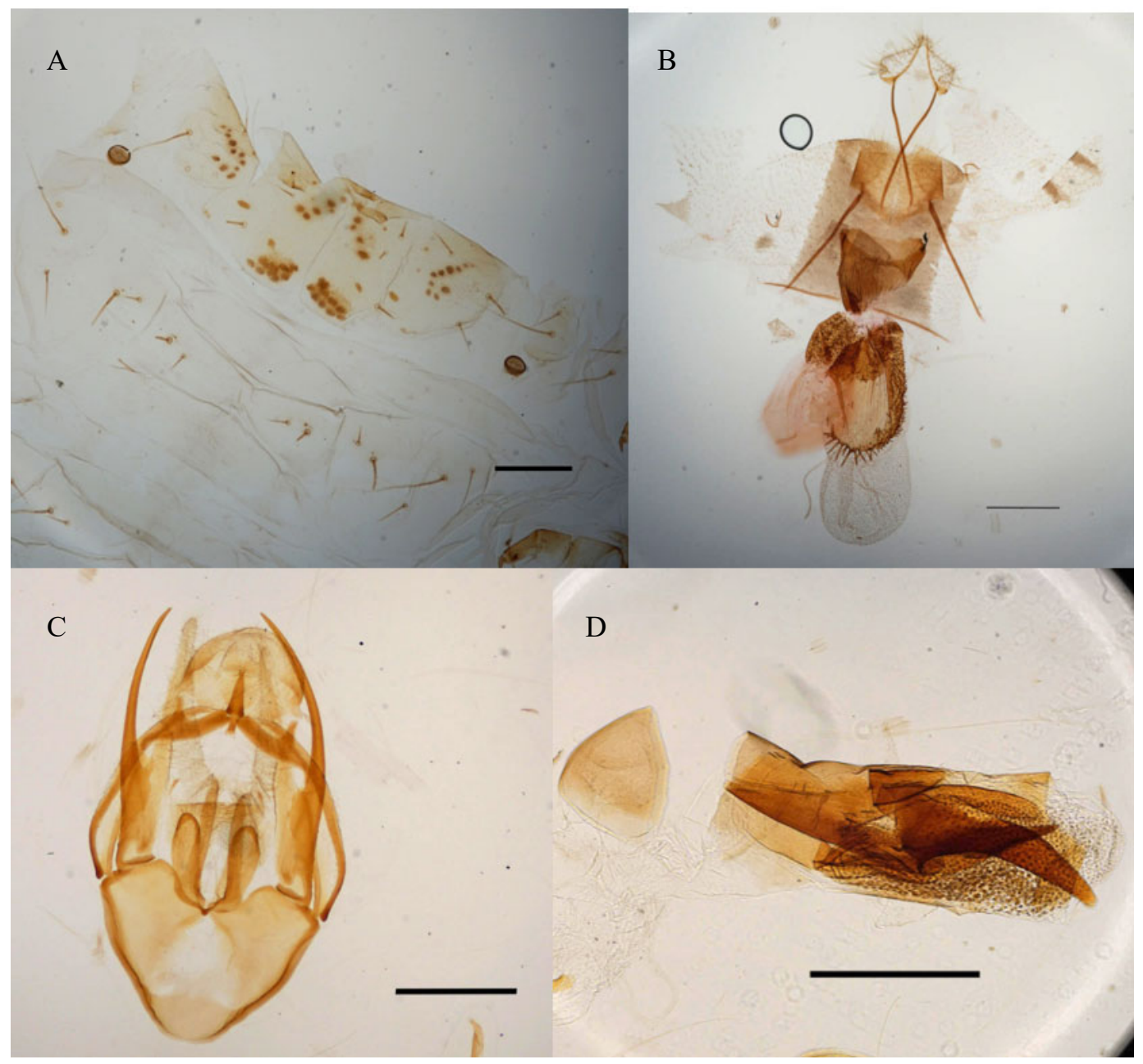

Fig. 3. Etiella zinckenella. A) Placa torácica de larva emergida desde cápsulas de Alstroemeria spp.; B) genitalia de macho, aedeagus removido; C) aedeagus; D) genitalia de hembra. Barras $=500 \mu \mathrm{m}$.

Fig. 3. Etiella zinckenella. A) Larval chest plate emerged from Alstroemeria spp. capsules; B) male genitalia, aedeagus removed; C) aedeagus; D) female genitalia. Bars $=500 \mu \mathrm{m}$.

León. 5 larvas colectadas, 2 hembras y 3 machos emergidos desde crianza en laboratorio.

\section{CONCLUSIÓN}

Esta es la primera detección e identificación de E. zinckenella afectando a especies nativas de la familia Alstromeriaceae en Chile.

\section{LITERATURA CITADA}

Artigas, J.N. 1994. Entomología Económica (Insectos de Interés Agrícola, Forestal, Médico y Veterinario (nativos, introducidos y susceptibles de ser introducidos). Editorial Universidad de Concepción 2:1-943.
Cepeda, D. 2017. Introducción a los Phycitinae de Chile (Lepidoptera: Pyralidae), nuevo registro y descripción de una nueva especie del género Homeographa Ragonot (1888). Insecta Mundi 0556:1-9.

Kuswantoro, H., M.S.Y.I. Bayu, Y. Baliadi, and W. Tengkano. 2017. Resistance of advanced soybean lines to pod borrer (Etiella zinckenella Treitschke). Biosaintifika. Journal of Biology \& Biology Education 9(2):317-324.

Munroe, E., and M. Solis. 1999. The Pyraloidea. p. 233-256. In N. Kristensen (ed.) Lepidoptera, Moths and Butterflies. Vol 1. Handbook of Zoology. Walter de Gruyter \& Co., Berlin, Germany. 
Prayogo, M., and M. Bayu. 2020. Validation of technology components for peanut pod borer (Etiella zinckenella Triet.) control. J. HPT Tropika 20(1):1-12.

Solis, M. 2006. Key to selec o selected Pyraloidea (Lepidoptera) larvae intercepted at U.S. ports of entry: Revision of Pyraloidea in "Keys to some frequently intercepted Lepidopterous larvae" by Weism 1986. USDA Systematic Entomology Laboratory. 1. ProceedingsEntomological Society of Washington 101(3).

Urra, F. 2018. Las polillas pirálidas. Santiago, Chile. Servicio Nacional del Patrimonio Cultural. Disponible en https://www. mnhn.gob.cl/613/w3-article-87533.html? noredirect=1. (Consulta 19 mayo 2019).
Whalley, P.E.S. 1973. The genus Etiella Zeller (Lepidoptera: Pyralidae): a zoogeographic and taxonomic study. Bulletin of the British Museum (Natural History) Entomology series, London, 28(1):1-21. XS

Zhang, X.Z. 2011. Damages caused by Etiella zinckenella to pigeon pea and its integrated control measures. Journal of Southern Agriculture 42(9):1066-1069. 